\title{
Statistical Texture Analysis-Based Approach for Fake Iris Detection Using Support Vector Machines
}

\author{
Xiaofu He, Shujuan An, and Pengfei Shi \\ Institute of Image Processing and Pattern Recognition, \\ Shanghai Jiao Tong University, Shanghai, 200240, China \\ \{xfhe,sj_an,pfshi\} asjtu.edu.cn
}

\begin{abstract}
This paper presents a novel statistical texture analysis based method for detecting fake iris. Four distinctive features based on gray level co-occurrence matrices (GLCM) and properties of statistical intensity values of image pixels are used. A support vector machine (SVM) is selected to characterize the distribution boundary, for it has good classification performance in high dimensional space. The proposed approach is privacy friendly and does not require additional hardware. The experimental results indicate the new approach to be a very promising technique for making iris recognition systems more robust against fake-iris-based spoofing attempts.
\end{abstract}

\section{Introduction}

Biometrics systems offer great benefits with respect to other authentication techniques, in particular, they are often more user friendly and can guarantee the physical presence of the user[1-2]. Iris recognition is one of the most reliable biometric technologies in terms of identification and verification performance. It mainly uses iris pattern to recognize and distinguish individuals since the pattern variability among different persons is enormous. In addition, as an internal (yet externally visible) organ of the eye, the iris is well protected from the environment and stable over time [3-9]. Whereas, it is important to understand that, as any other authentication technique, iris recognition is not totally spoof-proof. The main potential threats for iris-based systems are [10-12]: 1) Eye image:Screen image,Photograph,Paper print,Video signal; 2)Artificial eye:Glass/plastic etc; 3) Natural eye(user):Forced use; 4) Capture/replay attacks: Eye image, IrisCode template; 5) Natural eye(impostor): Eye removed from body, Printed contact lens. Recently, the feasibility of the last type of attack has been reported by some researchers [10-14]: they showed that it is actually possible to spoof some iris recognition systems with well-made iris color lens. Therefore, it is important to detect the fake iris as much as possible before subsequent iris recognition.

In previous research, Daugman introduced the method of using FFT (Fast Fourier Transform) in order to check the printed iris pattern [10-12]. His method detects the high frequency spectral magnitude in the frequency domain, which can be shown distinctly and periodically from the print iris pattern because of the characteristics of the periodic dot printing. However, if the input counterfeit iris is defocused and blurred purposely, the counterfeit iris may be accepted as live one. Some iris camera 
manufacturer also proposed counterfeit iris detection method by using the method of turning on/off illuminator and checking the specular reflection on a cornea. Whereas, such method can be easily spoofed by using the printed iris image with cutting off the printed pupil region and seeing through by attacker's eye, which can make corneal specular reflection [13]. Lee et al. [14] proposed a new method of detecting fake iris attack based on the Purkinje image by using collimated IR-LED (Infra-Red Light Emitting Diode). Especially, they calculated the theoretical positions and distances between the Purkinje images based on the human eye model. However, this method requires additional hardware and need the user's full cooperation. To some extent, this interactive mode demands cooperation of the user who needs to be trained in advance and will eventually increase the time of iris recognition.

In this paper, we propose a new statistical texture analysis based method for detecting fake iris. Four distinctive features based on co-occurrence matrix and properties of statistical intensity values of image pixels are used. A support vector machine (SVM) is used to characterize the distribution boundary, for it has good classification performance in high dimensional space and it is originally developed for two-class problems. The proposed approach is privacy friendly and does not require additional hardware. The remainder of this paper is organized as follows: the proposed method is described in section 2. Section 3 reports experiments and results. Section 4 concludes this paper.

\section{Proposed Approach}

\subsection{Preprocessing}

In our experiments, we find that the outer portion of the color contact lens (corresponding to regions closer to outer circle) provides the most useful texture information for fake iris detection since this section of the fake iris is insensitive to the pupil dilation. In addition, the iris maybe corrupted by the occlusion of eyelashes and eyelids, it is necessary to exclude them as much as possible. Therefore, we extract features only in the lower half part of the iris pattern (called region of interest, ROI), i.e we minimize the influence of eyelashes and eyelids by abandoning up half part of the iris pattern.

As mentioned above, useful iris information distributes in the outer portion of the color contact lens. We have found empirically that ROI is usually concentric with the outer circle of iris and the radius of the ROI areas is restricted in a certain range. We therefore detect outer circle to acquire the most discriminating iris features. Outer boundary is detected using Hough transform together with the improved Canny edge detector [15-17], shown in Fig. 1(a). After outer boundary detection, ROI is estimated according to the radius $r_{R O I}$ empirically, as seen in Fig.1(b). In order to achieve invariance to translation and scale, the ROI is further normalized to a rectangular block of a fixed size $W \times H$ by anti-clockwise unwrapping the iris ring, as shown in Fig. 1(c). 


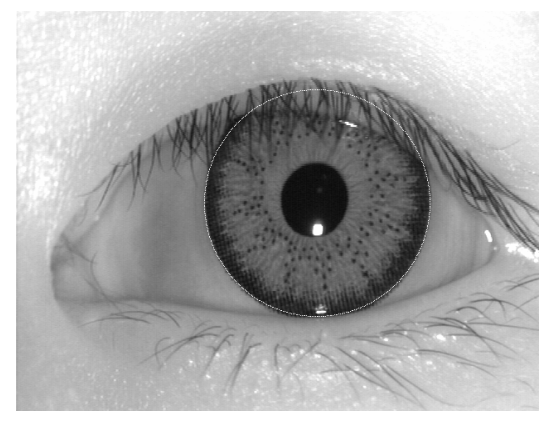

(a)

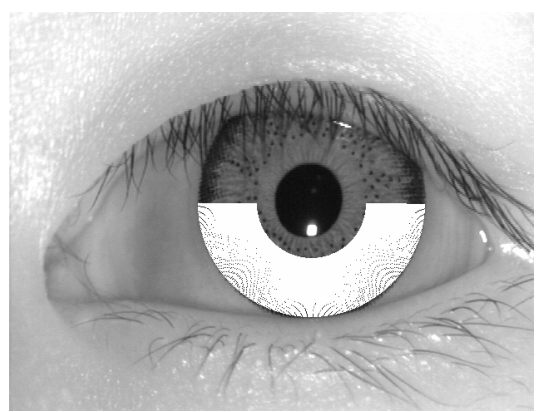

(b)

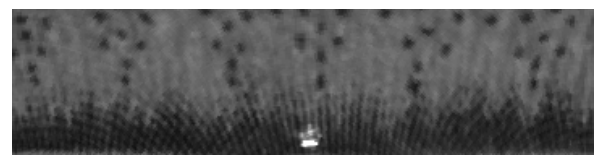

(c)

Fig. 1. Preprocessing. (a) Source color contact lens image and outer boundary detection result. (b) The normalization region used for fake detection. (c) Normalized image.

\subsection{Feature Vector}

The gray level co-occurrence matrices (GLCM) based analysis [18] is one of the most prominent approaches used to extract textural features. Each element $P(i, j)$ of the GLCM represents the relative frequency with which two neighboring pixels separated by a distance of certain columns and certain lines occur, one with gray tone $i$ and the other with gray tone $j$. Such matrices of gray-level spatial-dependence frequencies are a function of the angular relationship between the neighboring resolution cells as well as function of the distance between them. It is common practice to utilize four well-known properties, i.e, contrast, correlation, angular second moment (is also known as energy) and homogeneity, of a GLCM as the co-occurrence matrix-based features. Although the approach was proposed more than 30 years ago, the properties still remain amongst the most popular and the most discriminative types of texture features [19-20].

In this study, two well-known properties of the GLCM, i.e contrast con and angular second moment $a s m$ are utilized for creating feature vector. In addition, the mean $m$ and standard deviation $\sigma_{1}$ value of the intensity values of image pixels are also used for feature value. These four feature values are defined as following:

$$
m=\frac{1}{W \times H} \sum_{x=1}^{H} \sum_{y=1}^{W} I(x, y)
$$




$$
\begin{gathered}
\sigma_{1}=\sqrt{\frac{1}{W \times H} \sum_{x=1}^{H} \sum_{y=1}^{W}(I(x, y)-m)^{2}} \\
\text { con }=\sum_{i=1}^{N} \sum_{j=1}^{N}(i-j)^{2} P(i, j) \\
\text { asm }=\sum_{i=1}^{N} \sum_{j=1}^{N} P(i, j)^{2}
\end{gathered}
$$

Where $I$ denotes the normalized iris image, $W$ is the width of the normalized iris image, $H$ is the height of the normalized iris image. $P$ is the co-occurrence matrix, $N$ denotes the dimension of the co-occurrence matrix.

Therefore, these feature values are arranged to form a four dimensional feature vector.

$$
V=\left[m, \sigma_{1}, \text { con }, \text { asm }\right]^{T}
$$

\subsection{Classification}

After feature extraction, ROI is represented as a feature vector of length four. The features extracted are used for classification by SVM [21] that appear to be a good candidate because of their ability to transform the learning task to the quadratic programming problem in high-dimensional spaces. In addition, SVM is originally developed for two-class problems.

In this paper, radial basis functions (RBF) kernel function is used as,

$$
K\left(x, x_{i}\right)=\exp \left\{-\frac{\left|\mathrm{x}-x_{i}\right|^{2}}{\sigma_{2}{ }^{2}}\right\}
$$

where, $\mathrm{X}_{\mathrm{i}}$ comprises the input features, and $\sigma_{2}$ is the standard deviation of the RBF kernel, which is three in our experiments.

The input to the SVM texture classifier comes from a feature vector of length four. This reduces the size of the feature vector and results in an improved generalization performance and classification speed. The sign of the SVM output then represents the class of the iris. For training, +1 was assigned to the live iris class and -1 to the fake iris class. As such, if the SVM output for an input pattern is positive, it is classified as live iris.

\section{Experimental Results}

In this work, experiment is performed in order to evaluate the performance of the proposed method, which is implemented using Matlab 7.0 on an Intel Pentium IV 3.0G processor PC with 512MB memory. We manually collect 2000 live iris images, 250 
contact color lens images. 1000 live iris images and 150 contact lens images are used for training and the rest for testing. The positive samples (the live iris images) come from the SJTU iris database version 3.0 (Iris database of Shanghai Jiao Tong University, version 3.0) which is created by using contactless iris capture device. The negative samples (the color contact lens image, bought at market) come from those images that captured at one session. The size of eye images is $640 \times 480$. The radius $r_{R O I}$ of the ROI is 70 . The size of each normalized iris image is $90 \times 360$. The iris feature vector consists of a feature vector of length four. Samples of the live and fake iris are shown in Fig. 2.

The main drawback of the GLCM approach is the large computer resources required. For example, for an 8-bit image (256 gray level), the co-occurrence matrix has 65536 elements. The number of gray levels determines the size of the GLCM. To reduce this problem, the number of gray levels is set to 8 gray levels when scaling the grayscale values in image. The parameters of RBF kernel function are set: upper bound is 10 , standard deviation is 3 . The correct classification rate is $100 \%$. The average execution time for feature extraction and classification (for testing) is $31.3 \mathrm{~ms}$ and 14.7 $\mathrm{ms}$ respectively. The results indicate that the proposed scheme should be feasible in practical applications.

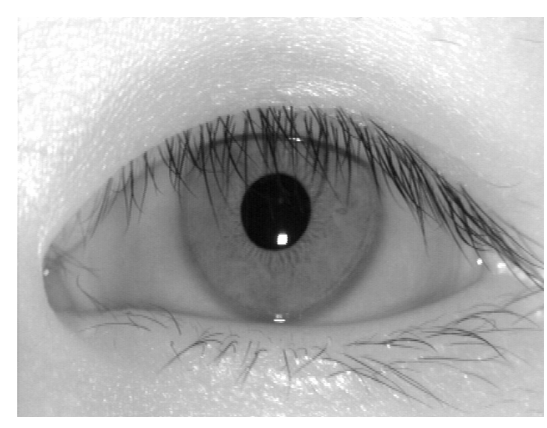

(a)

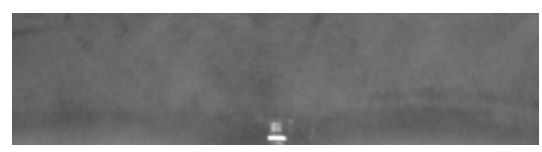

(c)

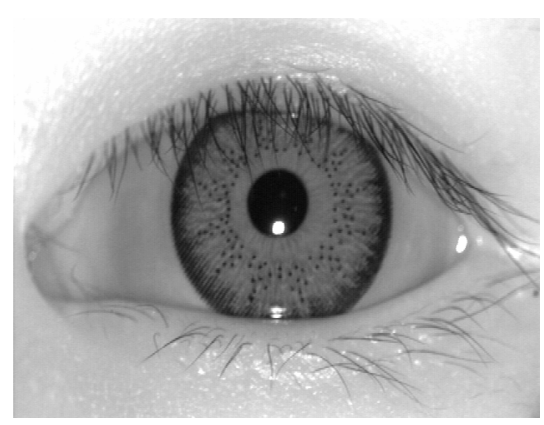

(b)

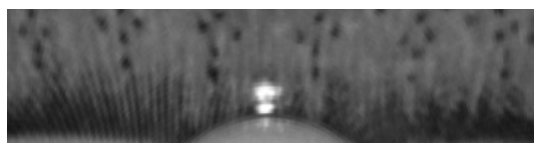

(d)

Fig.2. The test examples of live and fake iris. (a) Live eye. (b) Live eye with a color contact lens. (c) Normalized image of ROI of the live eye. (d) Normalized image of ROI of the live eye with a color contact lens.

\section{Conclusion}

In this paper, we have presented an efficient fake iris detection method based on statistical texture analysis. Four feature values, i.e the mean and standard deviation value of the intensity values of image pixels, contrast and angular second moment of 
the GLCM, are used for creating feature vector. We choose SVM to characterize the distribution boundary, for it has good classification performance in high dimensional space. Experimental results have illustrated the encouraging performance of the current method in accuracy and speed. The correct classification rate was $100 \%$. The average execution time for feature extraction and classification is $31.3 \mathrm{~ms}$ and $14.7 \mathrm{~ms}$ respectively using Matlab 7.0 on an Intel Pentium IV 3.0G processor PC with 512MB memory.

In the future work, we will extend the fake iris database and conduct experiments on a large number of iris databases in various environments for the proposed method to be more stable and reliable.

\section{Acknowledgements}

The authors would like to thank Mr. Eui Chul Lee (Dept. of Computer Science, Sangmyung University) for his helpful discussions. They also thank the anonymous referees for their constructive comments. This work is funded by the National Natural Science Foundation (No.60427002) and the National 863 Program of China (Grant No. 2006AA01Z119).

\section{References}

1. Jain, A.K., Bolle, R.M., Pankanti, S. (eds.): Biometrics: Personal Identification in Networked Society. Kluwer, Norwell, MA (1999)

2. Zhang, D.: AutomatedBiometrics: Technologies andSystems. Kluwer, Norwell, MA (2000)

3. Daugman, J.: High confidence visual recognition of persons by a test of statistical independence. IEEE Trans. Pattern Anal. Mach. Intell. 15(11), 1148-1161 (1993)

4. Daugman, J.: The importance of being random: Statistical principles of iris recognition. Pattern Recognition 36(2), 279-291 (2003)

5. Daugman, J.: How iris recognition works. IEEE Trans. on Circuits and Systems for Video Technology 14(1), 21-30 (2004)

6. Wildes, R.P.: Iris recognition: An emerging biometric technology. Proc. IEEE 85(9), 1348-1363 (1997)

7. Ma, L., Tan, T., Wang, Y., Zhang, D.: Personal identification based on iris texture analysis. IEEE Trans. Pattern Anal. Mach. Intell. 25(12), 1519-1533 (2003)

8. Sun, Z., Wang, Y., Tan, T., Cui, J.: Improving iris recognition accuracy via cascaded classifiers. IEEE Trans. on Systems, Man and Cybernetics, Part C 35(3), 435-441 (2005)

9. Park, K.R., Kim, J.: A real-time focusing algorithm for iris recognition camera. IEEE Trans. on Systems, Man and Cybernetics, Part C 35(3), 441-444 (2005)

10. Daugman, J.: Recognizing Persons by their Iris Patterns: Countermeasures against Subterfuge. In: Jain, et al. (eds.) Biometrics. Personal Identification in a Networked Society, pp. 103-121 (1999)

11. Daugman, J.: Demodulation by complex-valued wavelets for stochastic pattern recognition. International Journal of Wavelets, Multiresolution, and Information Processing 1(1), 1-17 (2003)

12. Daugman, J.: Iris Recognition and Anti-Spoofing Countermeasures. In: 7th International Biometrics Conference, London (2004)

13. http://www.heise.de/ct/english/02/11/114/ 
14. Lee, E.C., Park, K.R., Kim, J.: Fake iris detection by using purkinje image. In: Zhang, D., Jain, A.K. (eds.) Advances in Biometrics. LNCS, vol. 3832, pp. 397-403. Springer, Heidelberg (2005)

15. He, X., Shi, P.: A Novel Iris Segmentation method for Hand-held Capture Device. In: Singh, S., Singh, M., Apte, C., Perner, P. (eds.) ICAPR 2005. LNCS, vol. 3687, pp. 479-485. Springer, Heidelberg (2005)

16. Fleck, M.M.: Some defects in finite-difference edge finders. IEEE Transactions on Pattern Analysis and Machine Intelligence 14(3), 337-345 (1992)

17. Canny, J.: A Computational Approach to Edge Detection. IEEE Transaction on Pattern Analysis and Machine Intelligence 8, 679-714 (1986)

18. Haralick, R.M., Shanmugam, K., Dinstein, I.: Textural features for image classification. IEEE Trans. Syst. Man Cybern. 3(6), 610-621 (1973)

19. Soares, J.V., Renno, C.D., Formaggio, A.R., etc.: An investigation of the selection of texture features for crop discrimination using SAR imagery. Remote Sensing of Environment 59(2), 234-247 (1997)

20. Walker, R.F., Jackway, P.T., Longstaff, D.: Genetic algorithm optimization of adaptive multi-scale GLCM features. Int. J. Pattern Recognition Artif. Intell. 17(1), 17-39 (2003)

21. Burges, C.J.C.: A tutorial on support vector machines for pattern recognition. Data Mining Knowledge Discovery 2, 955-974 (1998) 